\title{
DISCUSSIONS
}

\section{Rowe's stress-dilatancy relation based on friction}

\author{
DE JOSSELIN DE JONG, G. (1976). Géotechnique 26, No. 3, 527-534.
}

\section{G. Gudehus, Karlsruhe University}

The argument of de Josselin de Jong concerning stress-dilatancy is certainly preferable to those of Rowe and Horne. The condition of maximum required friction in the actual instantaneous direction of slip is in fact a property of a slip mechanism, as was discovered by Coulomb. It appears advisable, however, to formulate some restrictions to the theory.

Firstly, the assumption of families of equally inclined toothed separation planes is at best kinematically acceptable for a cuboidal sample under plane strain. Still, this is merely an assumption, but it appears more realistic than for cylindrical compression.

Secondly, $D=-\dot{\epsilon}_{2} / \dot{\epsilon}_{1}$ is not generally a function of $R=\sigma_{1} / \sigma_{2}$ and the previous history. This can be the case for a continued rectangular deformation, but not for a continuation of deformation with different principal axes of stress and strain rate tensors. If the principal axes deviate by a certain angle, $\alpha, D$ is also a function of $\alpha$ (Vardoulakis, 1977). Therefore, one should restrict the theory to coaxial stress and strain rate tensors. $D$ can also be looked upon as an instantaneous kinematical constraint in the sense of Dietrich (1977) depending on the previous history and not only on $R$. Thus, $E=R / D$ cannot, in general, be assumed as a constant from the very beginning.

Now, the analysis can follow the lines of de Josselin de Jong, keeping in mind the above restrictions. Taking the generalized Coulomb's principle as a defining property of a slip mechanism, the conclusion can be formulated as follows: for a sample of a rigid granular material under continued plane rectangular strain the assumption of equally inclined toothed slip planes leads to a constant $R / D=\tan ^{2}\left(\pi / 4+\phi_{\mu} / 2\right)$.

\section{Author's reply}

It pleases me that Gudehus opens his discussion by agreeing with the friction analysis for developing Rowe's stress-dilatancy relation. The restrictions proposed subsequently are due to the character of the model adopted in the analysis. The objective of the article was not to consider the adequacy of the model in representing the behaviour of granular media, but to show how to treat such a model consistently, once adopted. In commenting on the remarks it is therefore necessary to enter into questions that were not mentioned in the article.

The first restriction is based on the consideration, that it is kinematically impossible for two intersecting shear planes to develop simultaneously. The four separated bodies cannot slide with respect to each other along those planes in a compatible manner, i.e. without creating gaps or overlaps. This is correct. However, there is no objection for sliding planes to develop subsequently. An example is shown by the photograph of the interior of a sand body (Fig. 1), containing a few percent of cement, sheared under constant stress, solidified and sawed afterwards. From the distortion of the coloured layers it is observed, that sliding along AA occurred first and that BB developed subsequently. The deformation of sand, where many grains slide with respect to each other is replaced in the model by discrete sliding planes with teeth. In the case of an axial symmetric stress distribution, the normals to potential sliding planes form a symmetric cone around the axis. Any combination of sliding planes is possible, if occurring subsequently. Therefore the model is not restricted to a cuboidal sample, but includes the cylindrical compression as well. 
Fig. 1. Sequence of sliding: AA first, BB afterwards

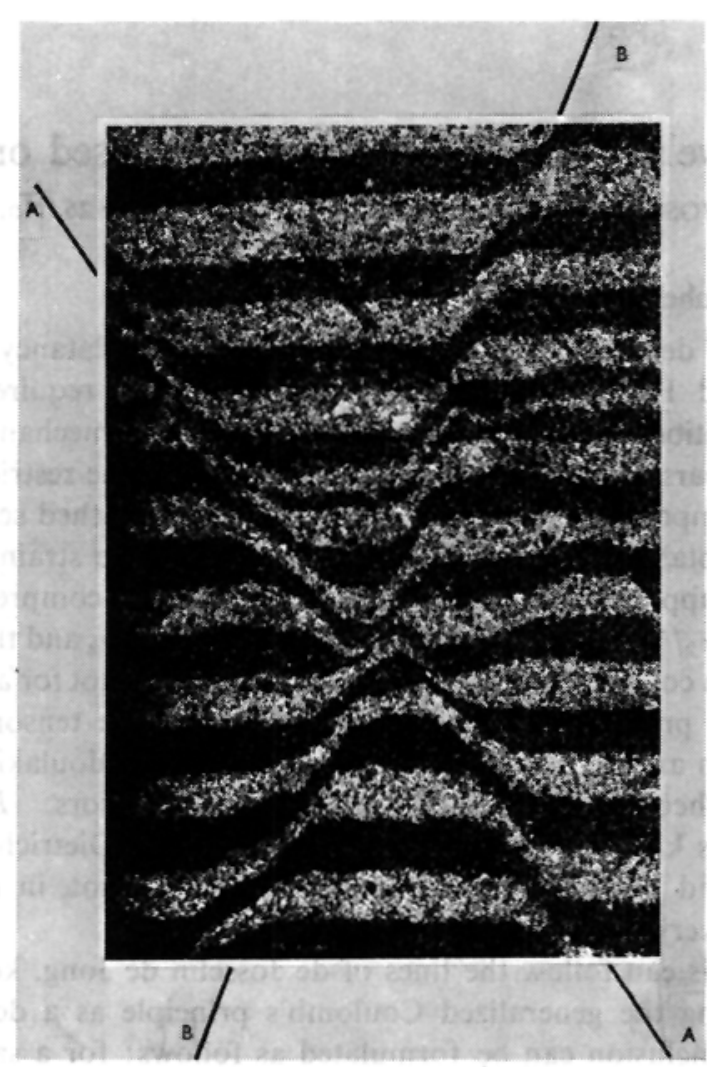

The second restriction deals with non-coaxiality. The situation considered in the Paper is a loading, such that the tensors of stress and increment of stress are coaxial, but that the strain rate tensor deviates from both of them. It can be shown (de Josselin de Jong, 1977) that in that case, the deviation angle never exceeds $\pm \frac{1}{2}\left(\phi^{*}-\theta\right)$, where $\frac{1}{2} \phi^{*}$ is the angle between the bisectrix of the principal stress and the sliding planes, and $\theta$ is the angle between teeth and sliding planes. The analysis in the Paper shows, that for that case $D^{*}$ is more relevant than $D$.

The model treated in the Paper is restricted to this case and cannot deal with the kind of non-coaxiality considered by Deitrich. His non-coaxiality is the deviation of strain rate tensor and stress tensor, when the tensor for the increment of stress is no more coaxial to the tensor of the ambient stress state. Then all kinds of deviation angles are possible, with a maximum of $\frac{1}{2} \pi$. Such non-coaxiality was observed when investigating the effect of stress reversals.

I agree with Gudehus, that the analysis is restricted to the case of coaxiality of stress and stress increment. In order to include also stress reversals and all other possible stress paths, other models are to be conceived. However, if an adequate model can be indicated, its analysis should be based on friction.

\section{REFERENCES}

De Josselin de Jong, G. (1977). Mathematical elaboration of the double sliding, free rotating model. To appears as: Engineering report No. 4/77 in Archwm Mech. Stosow.

Dietrich, Th. (1977). A comprehensive mechanical model of sand at low stress level. Submitted to Speciality Session, Ninth Int. Conf. Soil Mech. Fdn Engng, Tokyo.

Vardoulakis, J. (1977). Equilibrium bifurcation on granular earth bodies. To appear in: Recent advances in the application of stability analysis in geotechnical problems. University of Waterloo. 